\title{
Cotton-wool spots, red-lesions and hard-exudates distinction using CNN enhancement and transfer learning
}

\author{
Sinan S. Mohammed Sheet ${ }^{1}$, Tian-Swee Tan ${ }^{2}$, M. A. As' ${ }^{3}{ }^{3}$, Wan Hazabbah Wan Hitam ${ }^{4}$, \\ Qi Zhe $\mathrm{Ngoo}^{5}$, Matthias Tiong Foh thye ${ }^{6}$, Kelvin Ling Chia hiik ${ }^{7}$ \\ ${ }^{1}$ Technical Engineering College of Mosul, Northern Technical University, Mosul, Iraq \\ ${ }_{1,2,3,6,7}$ School of Biomedical Engineering \&Health Sciences, Faculty of Engineering, Universiti Teknologi Malaysia \\ (UTM), UTM Johor Bahru, Johor, Malaysia \\ ${ }^{4,5}$ Department of Ophthalmology and Visual Sciences, School of Medical Sciences, Health Campus, Universiti Sains \\ Malaysia (USM), Kota Bharu Kelantan, Malaysia
}

\begin{tabular}{l}
\hline \hline Article Info \\
\hline Article history: \\
Received May 4, 2021 \\
Revised Jul 13, 2021 \\
Accepted Jul 18, 2021 \\
\hline
\end{tabular}

\section{Keywords:}

Accurracy

CLAHE Filter

$\mathrm{CNN}$

Image enhancement

Retinal disease

\begin{abstract}
The automatic retinal disease diagnosis by artificial intelligent is an interesting and challenging topic in the medical field. It requires an appropriate image enhancement technique and a sufficient training dataset for the specific retina conditions. The aim of this study was to design an automatic diagnosis convolutional neural network (CNN) model which does not require a large training dataset to specifically identify diabetic retinopathy symptoms, which are cotton wool, exudates spots, and red lesion in colour fundus pictures. A novel framework comprised image enhancement method by using upgraded contrast limited adaptive histogram equalization (UCLAHE) filter and transferred pre-trained networks was developed to classify the retinal diseases regarding to the symptoms. The performance of the proposed framework was evaluated based on accuracy, sensitivity, and specificity metrics. The collected results have proven the robustness of the proposed framework in offering good accuracy in retina diseases diagnosis.
\end{abstract}

This is an open access article under the CC BY-SA license.

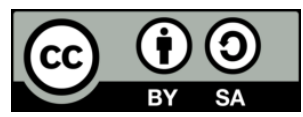

\section{Corresponding Author:}

Tian-Swee Tan

BioInspired Device and Tissue Engineering Research Group

School of Biomedical Engineering and Health Sciences, Universiti Teknologi Malaysia

81310 UTM Johor Bahru, Johor, Malaysia

Email: tantswee@utm.my

\section{INTRODUCTION}

Diabetic retinopathy is one of the main reasons that lead to vision loss for diabetic patients. Automatic retinal disease diagnosis is in demand to detect its symptoms in the early stage to prevent blindness [1], [2]. According to [3], there were 0.4 million and 2.6 million patients who were partially blind and had critical vision weakness respectively due to diabetic retinopathy in 2015. At the beginning of the retina ailment, retina abnormality, for instance, observable retina in the fundus pictures, signifying the deviations in the eye vision. The automatic detection of retina abnormalities offers an active method to gain a prompt judgement of diabetic retinopathy [4].

Diabetic retinopathy can be categorized into several stages: mild, moderate, and severe [5]. The diseases are classified based on the types of retina disorder, including hard exudates, cotton wool spots and red lesion. These are the common symptoms that indicate retinopathy in the eye. To obtain an accurate diagnosis, a detection model is required to identify and also differentiate these symptoms from each other [3] since the result may lead to different diagnostic status and implications [6]. Another novel enhancement 
approach to boost the edge detection performance an eight-directional filters were used for normalizing intensity and maximize the edge magnitude for Mini Kirsch diagnosis [7].

Moving from human inspection to machine inspection will impose a new approach in the medical field to analyze the multilane classification and the disease conditions [8]. The strategy of implementing deep learning (DL) approach for the disease recognition and image distinction requires a primary training set which is annotated with those lesion symptoms. The most common technique used in the eye imaging field is the funduscopic method due to its simplicity to be handled, widely reachable, and suitability for documentation [9]-[11]. It is possible to further process those fundus pictures based on the desirable features through adjustment in spatial domain.

Orlando et al. had presented a remarkable indication on selecting the suitable image pre-handling method before they fed the dataset to convolutional neural network (CNN) [12]. The contrast limited adaptive histogram equalization (CLAHE) filter shows a great performance to enhance and remove the noise in eye retina images [13]-[15]. However, it has a drawback on its predefined clip limit that causes some irregular illuminance and excessive artifacts in the images [16]. On the other hand, deep transfer learning (DTL) approach, which uses pre-trained CNN dataset, appears to be a great solution to overcome the dataset shortage problem. In the pre-trained model, the universal pictures from "Image-Net" can be used as a primary weightage values in the DTL approach [17].

Several studies had presented several methods to detect the diabetic retinopathy of different conditions, either utilizing an extractor to obtain the diseases features and fed it to support vector machine (SVM) classifier, or applying a combined pre-trained model to optimize the output classification [18]-[20]. Most of these studies implemented multiple segmentation techniques to determine the region of interest (ROI), and then input the segmented dataset to pre-trained models. However, the output results prone to experience disease information lost, such as disease symptoms. In addition, the studies that used the unrefined fundus pictures resulted in a low accuracy classification output. Therefore, a pre-processing stage is required to improve the image appearance, and eventually reducing the risk of information loss to secure a better disease recognition [21], [22]. The purpose of this study was to propose a DL model with great performance and a good accuracy to distinguish the exudates, cotton-wool spots and red lesion in fundus picture.

The study utilized the sample pictures of diabetic patients which are obtained from "Fishare" website that covers 169 and 98 fundus pictures that are bright and red lesion abnormalities respectively. The bright lesion is caused by the exudates and cotton wool spots. The assessment and verification of the proposed method are also carried and the framework performance is compared with the previous studies.

\section{RESEARCH METHOD}

Overall, the pre-trained dataset from deep CNN transfer learning was used for the detections of exudates, cotton-wool spots, and red lesion in fundus pictures. The DTL method is preferred as it does not require big quantities of image samples [23]. Figure 1 illustrates the process of the proposed method.

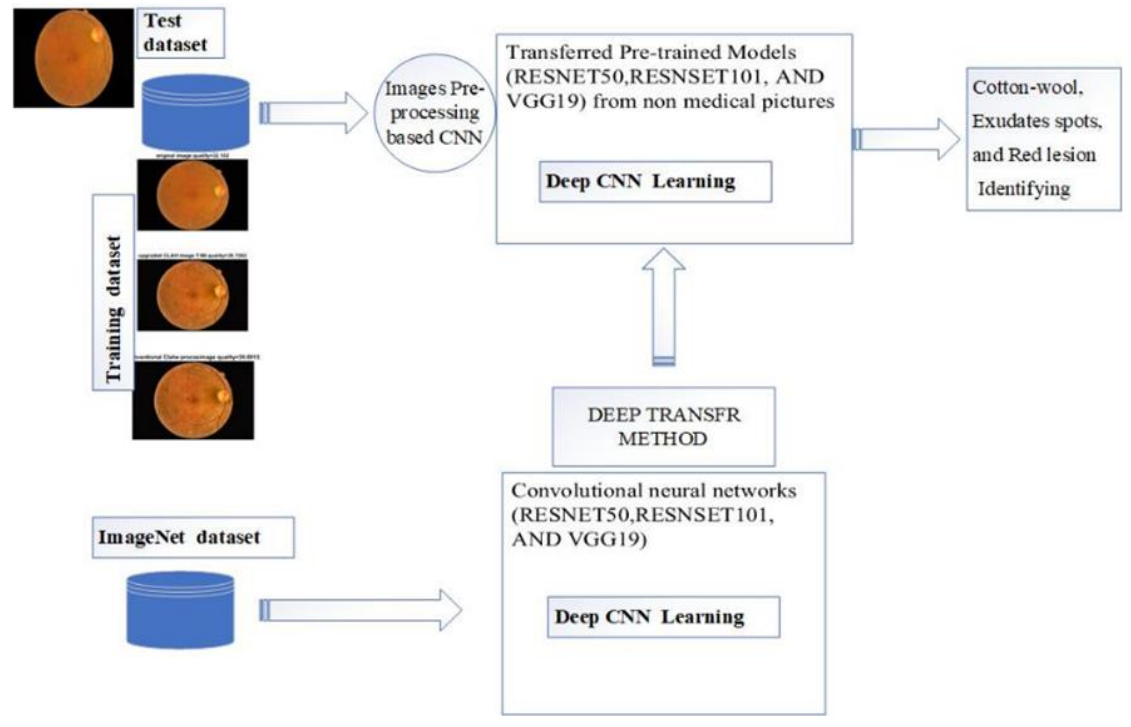

Figure 1. Workflow of exudates, cotton-wool, and red lesion classification using deep CNN enhancement and transfer learning 
Several image processing approaches were conducted on the fundus picture before feeding it into the pre-trained network [24]. The CNN was trained intently to extract the important image features for enhancement. The whole fundus image was fed into the pre-train model without segmentation to obtain the calculation of the whole features where using segmentation method to detect the retina abnormalities my leading to losing important information, as an example, the following study used segmentation to focus on a specific region and ignored the rest area of the retina which may have very useful syndromes [22], [25].

\subsection{Image dataset}

Initially, there are a total of 617 fundus pictures, downloaded from the Fishare website, that are provided by the ophthalmologists for clinical analysis. After examining all those pictures, there are 90, 79 and 98 pictures that contain the cotton wool, exudates spots and red lesion selected respectively [26]. Meanwhile, $70 \%$ of the images for each category are used for CNN training purpose while the rest of the images are used for Test purpose.

\subsection{Image pre-processing}

Fundus pictures may have some defects owing to the retinal leaning, inaccurate and blurred spot due to limitation of acquiring devices, and certain unexpected circumstances that lead to miscellaneous poorquality pictures. It is significant to improve the picture quality to improve the performance and accuracy of CNN classification model.

\subsubsection{Upgraded CLAHE}

The upgraded CLAHE filter, also known as the enhanced version of conventional CLAHE was picked according to its ability to produce the best enhancement output among the existing enhancement methods. The fixed clip limit was replaced with a global threshold that is adaptive to the gray level of the pictures. The conventional and upgraded CLAHE equations are denoted as in (1) and (2) [16]:

$$
\begin{aligned}
& \text { CLIP LIMIT }=\left[\frac{\varphi}{L}\right]+\left[\beta \cdot\left(\varphi-\left[\frac{\varphi}{L}\right]\right)\right] \\
& \text { CLIP LIMIT }=\frac{T}{80}
\end{aligned}
$$

Where $\mathrm{T}$ is the global threshold, $\varphi$ is the pixels population in each block, $\beta$ is the clip factor, and $\mathrm{L}$ is the grayscale of the image. Figure 2(a)-(c) shows an example of the original fundus images and the output images that have been processed with conventional and upgraded CLAHE filters.

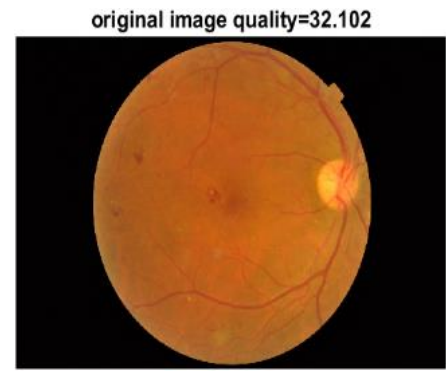

(a)

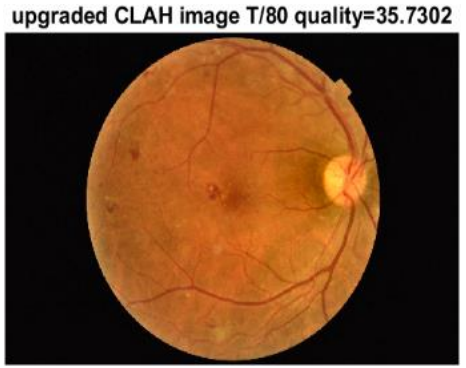

(b)

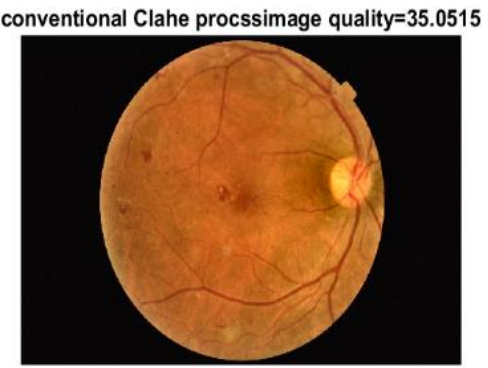

(c)

Figure 2. Example of; (a) original fundus image; (b) output image from upgraded CLAHE filter; (c) the output image from conventional CLAHE filter

\subsubsection{CNN Enhancement step}

In this section the single $\mathrm{CNN}$ has been developed for using it as test device for give the last decision for enhancement the fundus images or not. Tables 1-3 show the test result, Tables present four phases, phase 1 for no enhancement, phase 2 for limit enhancement on single disease case, in a Table can be seen that "0" for non-enhancement, while " 1 " for applying the enhancement. Based on the supreme results of these Tables the enhancement topology has been chosen. 
Table 1. CNN enhancement decision truth Table for cotton-wool spots

\begin{tabular}{ccccccc}
\hline Preparing method & \multicolumn{7}{c}{ (Class labels) Cotton-wool Spots } \\
No enhancement=0 & \multicolumn{2}{c}{ Sigle CNN1 } & & \multicolumn{1}{c}{ Sigle CNN2 } & \\
Using MCLAHE=1 & Cotton-wool Spots & Red Lesions & Result & Cotton-wool Spots & Hard Exudates & Result \\
\hline Phase 1 & 0 & 0 & 80.4 & 0 & 0 & 84.3 \\
Phase2 & 0 & 1 & 94.6 & 0 & 1 & 90.2 \\
Phase3 & $\mathbf{1}$ & 0 & $\mathbf{1 0 0}$ & $\mathbf{1}$ & 0 & $\mathbf{9 5 . 7}$ \\
Phase4 & $\mathbf{1}$ & 1 & $\mathbf{1 0 0}$ & 1 & 1 & 93.5 \\
\hline
\end{tabular}

Table 2. CNN enhancement decision truth Table for red lesions

\begin{tabular}{ccccccc}
\hline Preparing method & \multicolumn{5}{c}{ (Class labels) Red Lesions } \\
No enhancement=0 & & Sigle CNN1 & Sigle CNN2 & \\
Using MCLAHE=1 & Red Lesions & Cotton-wool Spots & Result & Red Lesions & Hard Exudates & Result \\
\hline Phase 1 & 0 & 0 & 80.4 & 0 & 0 & 20.8 \\
Phase2 & $\mathbf{0}$ & 1 & $\mathbf{1 0 0}$ & 0 & 1 & 86.6 \\
Phase3 & 1 & 0 & 94.6 & $\mathbf{1}$ & 0 & $\mathbf{9 6 . 2}$ \\
Phase4 & $\mathbf{1}$ & 1 & $\mathbf{1 0 0}$ & 1 & 1 & 24.5 \\
\hline
\end{tabular}

Table 3. CNN enhancement decision truth Table for hard exudates

\begin{tabular}{|c|c|c|c|c|c|c|}
\hline \multirow{3}{*}{$\begin{array}{l}\text { Preparing method } \\
\text { No enhancement }=0 \\
\text { Using MCLAHE }=1\end{array}$} & \multicolumn{6}{|c|}{ (Class labels) Hard Exudates } \\
\hline & \multicolumn{3}{|c|}{ Sigle CNN1 } & \multicolumn{3}{|c|}{ Sigle CNN2 } \\
\hline & Hard Exudates & Cotton-wool Spots & Result & Hard Exudates & Red Lesions & Result \\
\hline Phase 1 & 0 & 0 & 84.3 & 0 & 0 & 20.8 \\
\hline Phase2 & $\mathbf{0}$ & 1 & 95.7 & $\mathbf{0}$ & 1 & 96.2 \\
\hline Phase3 & 1 & 0 & 90.2 & 1 & 0 & 86.6 \\
\hline Phase4 & 1 & 1 & 93.5 & 1 & 1 & 24.5 \\
\hline
\end{tabular}

Finally, from Tables 1-3, the best performance can be obtained when the cotton-wool spots images are processed with optimized CLAHE filter, while the red lesions can be processed with either upgraded filter processing or without can be obtained higher performance. Lastly, the hard exudates can be input into classification model without needing for enhancement process to get the best performance.

\subsection{Augmentation}

In order to overcome the problem of dataset shortage for each category, the augmentation method is applied. The pictures are rotated with a step size of 30 degrees ranging from $0^{\circ}$ to $330^{\circ}$. This allows the introduction of more pictures feature to improve the model generalization and reduce the tendency of overfitting. The augmentations of dataset are shown in Figure 3(a)-(d).

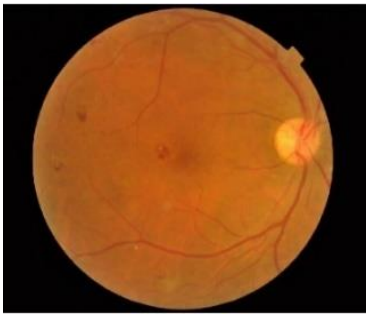

(a)

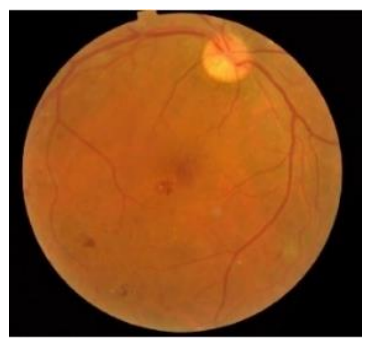

(c)

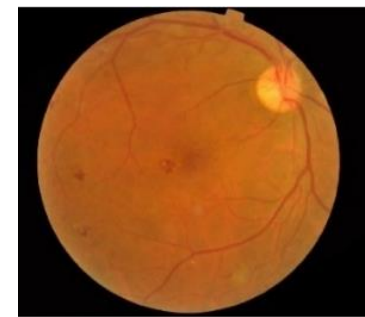

(b)

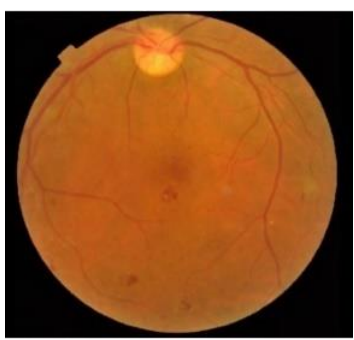

(d)

Figure 3. Result of augmentation method for rotation of; (a) $0^{\circ}$; (b) $30^{\circ}$; (c) $60^{\circ}$; and (d) $90^{\circ}$ 


\subsection{Deep transfer learning}

A pre-trained network was chosen based on its ability to distinguish multiclass of fundus pictures samples [27]. However, the final network layer, which is able to classify the three types of aforementioned diabetic retinopathy symptoms, is added to the pre-trained network. This approach could shorten the training time instead of training the new network from scratch. The selected pre-trained models include RESNET50, RESNET101, and VGG19, which have been used in the previous studies for the analysis, recognition and classification of medical images and signals [28]. Figure 4 shows one of the results from VGG19 model where there is exudate detected as labelled in the Figure.

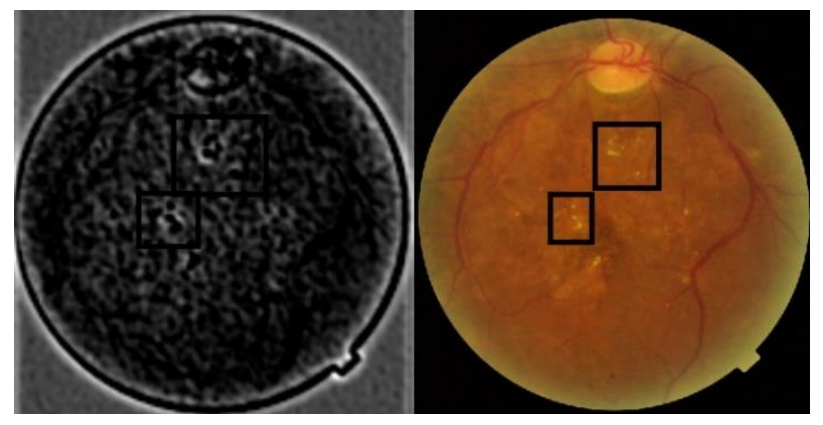

Figure 4. Detected exudate in fundus picture in VGG19 model

\section{RESULTS AND DISCUSSION}

Image enhancement is important to highlight the desired feature to improve the classification performance [10], [21], [29], [30]. In this study, a revised version of CLAHE filter was applied to improve the picture quality and to emphasize the fine vessels in the retinal pictures. Moreover, a novel step was introduced where a single CNN model is employed to measure the quality of the enhancement dataset based on optimist output CNN classification accuracy.

At the end of the image pre-processing stage, the dataset was fed to a few deep transfer pre-trained networks which were RESNET50, RESNET101, and VGG19 model. Table 4 shows the results obtained using the conventional and upgraded CLAHE filters for image enhancement for the three CNN models. CNN models which use the input enhanced image from upgraded CLAHE filter show a better classification accuracy than those of conventional CLAHE filter. It is consistent with the result of primary single CNN enhancement but not with the elective mode method.

Table 4. Comparison of image classification accuracy among different CNN models for input images enhanced by conventional and upgraded CLAHE filter

\begin{tabular}{lcc}
\hline Pretrained CNN model & Conventional CLAHE & Upgraded CLAHE \\
\hline RESNET50 & $56 \%$ & $56 \%$ \\
RESNET101 & $57.57 \%$ & $59.1 \%$ \\
VGG19 & $57.57 \%$ & $60 \%$ \\
\hline
\end{tabular}

The upgraded CLAHE filter, which is modelled by the primary single CNN model, exhibited noTable improvement based on the elective enhancement approach (decision truth Table) that can be seen in Table 5. The elective enhancement procedure has improved the output classification for all three pre-trained models. The classification accuracy for RESNET50 model shows consistent performance for different input datasets, and has the lowest accuracy 97\%, among the CNN models as shown in Figure 5. Next, to elevate the performance of RESNET50 model, an augmentation approach was used to increase the variation of the training data set. Figure 6 shows the better classification result accuracy after the implementation of the augmentation method, typically in hard exudates detection.

Table 5. Performance metric Table for each CNN models

\begin{tabular}{lccc}
\hline Pretrained model & Accuracy $(\%)$ & Sensitivity $(\%)$ & Specificity $(\%)$ \\
\hline RESNET50 & 100 & 100 & 100 \\
RESNET101 & 100 & 100 & 100 \\
VGG19 & 100 & 100 & 100 \\
\hline
\end{tabular}




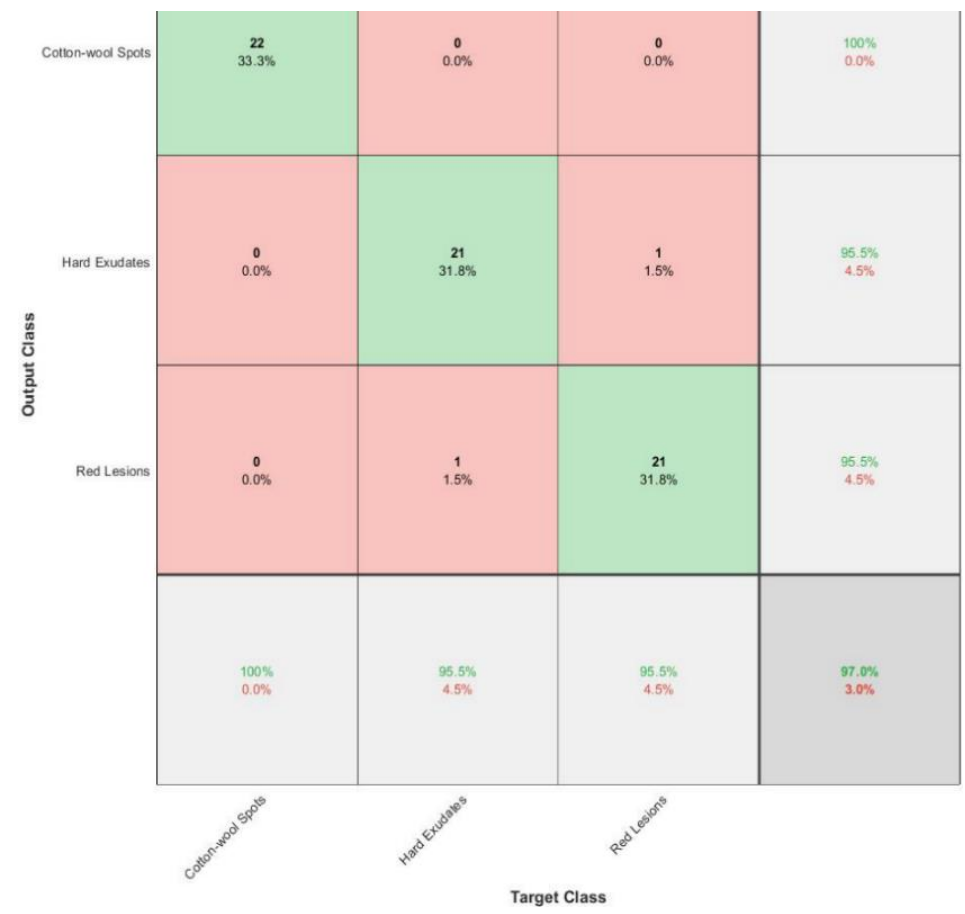

Figure 5. Confusion matrix of RESNET50 model

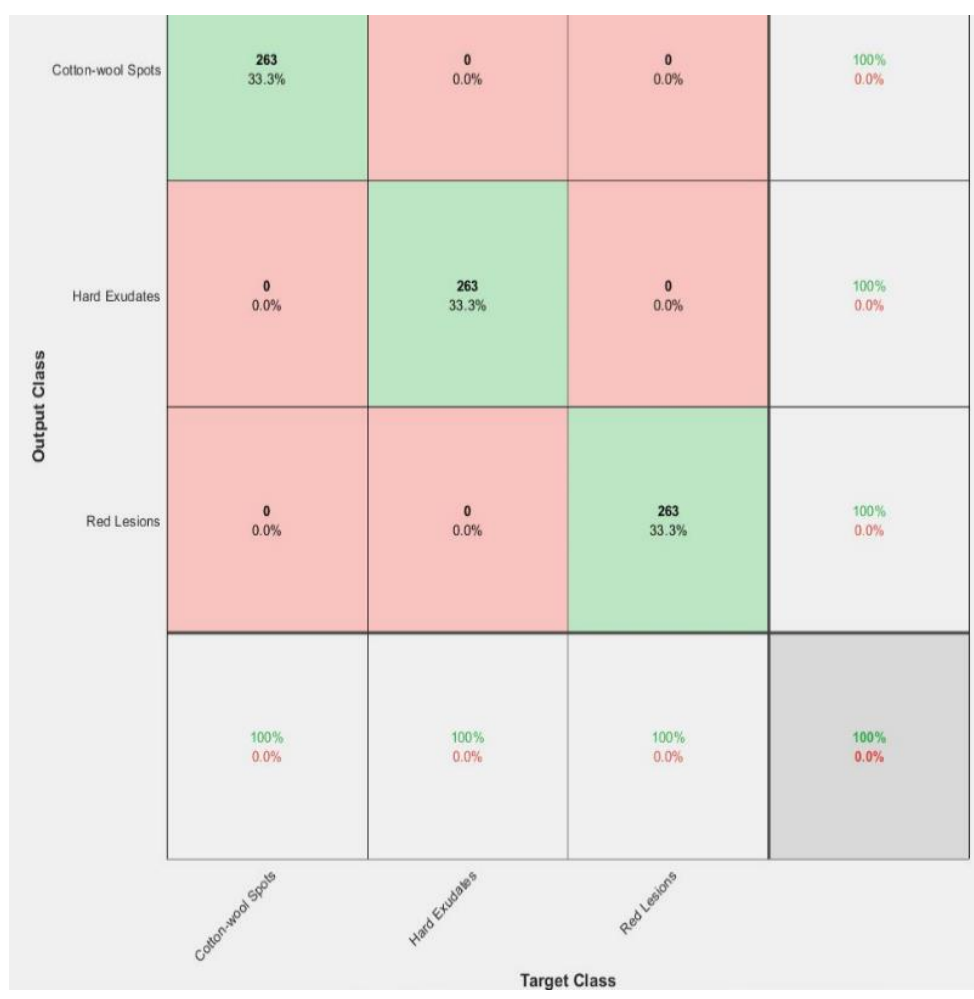

Figure 6. Confusion matrix of RESNET50 after the implementation of augmentation preprocessing

Performance analysis to determine the modelling uncertainties was carried out, based on the classification accuracy (ACC), sensitivity (Sen), and specificity (Spe). The equations for these three parameters are expressed as follows [4]: 


$$
\begin{aligned}
& A C C=\frac{\left(T_{N}+T_{P}\right)}{\left(T_{N}+F_{P}+T_{P}+F_{N}\right)} \\
& \text { Sen }=\frac{T_{P}}{\left(T_{P}+T_{N}\right)} \\
& \text { Spe }=\frac{T_{N}}{\left(T_{N}+F_{P}\right)}
\end{aligned}
$$

Where TP is the rate of true classified, TN is the rate of true negative classified, FP is the false sample classified, and FN is the false negative classified.

Table 5 presents the calculated performance metrics for each pre-trained CNN model. Nevertheless, these results suggest that data obtained using elective enhancement and deep learning transfer approach has optimized the accuracy of diabetic retinopathy symptoms detection by highlighting the important features in the image. Comparing the result with a previous study [31] that used automated machine learning, the proposed method in this study shows consistent and higher performance metrics as shown in Table 6 .

Table 6. Comparison Table with the previous study

\begin{tabular}{lcc}
\hline Retinopathy condition & $\begin{array}{c}\text { Proposed method } \\
\text { (sensitivity/specificity) }\end{array}$ & $\begin{array}{c}\text { Previous study [31] } \\
\text { (sensitivity/specificity) }\end{array}$ \\
\hline Exudates & $100 / 100$ & $0.95 / 0.86$ \\
Cotton-wool spots & $100 / 100$ & $0.70 / 0.93$ \\
All bright lesions & $100 / 100$ & $0.95 / 0.88$ \\
\hline
\end{tabular}

\section{CONCLUSION}

CNN enhanced and transfer learning method was proposed for detecting diabetic retinopathy symptoms such as hard exudates, cotton wool spots and red lesion. This approach potentially assists the retinal specialists to analyze and automatically classify the fundus picture of diabetic patients regarding the symptoms. Results show that the proposed method has a better classification accuracy compared to the previous study. Furthermore, the use of a deep transfer learning approach could reduce the dataset needed to train the network. For future development, more fundus pictures with other retinal diseases would be input to the network for wider applications. Provide a statement that what is expected, as stated in the "Introduction" chapter can ultimately result in the "Results and Discussion" chapter, so there is compatibility. Moreover, it can also be added the prospect of the development of research results and application prospects of further studies into the next (based on result and discussion).

\section{ACKNOWLEDGEMENTS}

The study was supported by Universiti Teknologi Malaysia and Fundamental Research Grant Scheme (FRGS), under the grant code R.J130000.7851.5F282.

\section{REFERENCES}

[1] M. D. Abràmoff, M. K. Garvin and M. Sonka, "Retinal Imaging and Image Analysis," in IEEE Reviews in Biomedical Engineering, vol. 3, pp. 169-208, 2010, doi: 10.1109/RBME.2010.2084567.

[2] A. Balasopoulou et al., "Symposium Recent advances and challenges in the management of retinoblastoma Globe - saving Treatments," BMC Ophthalmology, vol. 17, no. 1, pp. 1-1, 2017.

[3] X. Zeng, H. Chen, Y. Luo and W. Ye, "Automated Diabetic Retinopathy Detection Based on Binocular SiameseLike Convolutional Neural Network," in IEEE Access, vol. 7, pp. 30744-30753, 2019, doi: 10.1109/ACCESS.2019.2903171.

[4] V. Chandore and S. Asati, "Automatic Detection of Diabetic Retinopathy using deep Convolutional Neural Network," International Journal of Advance Research, Ideas and Innovations in Technology, vol. 3, pp. 633-641, 2017.

[5] M. H. A. Fadzil, L. I. Izhar, H. Nugroho and H. A. Nugroho, "Analysis of retinal fundus images for grading of diabetic retinopathy severity," Medical \& Biological Engineering \& Computing, vol. 49, no. 6, pp. 693-700, Jun 2011, doi: 10.1007/s11517-011-0734-2.

[6] K. He, X. Zhang, S. Ren and J. Sun, "Deep Residual Learning for Image Recognition," 2016 IEEE Conference on Computer Vision and Pattern Recognition (CVPR), 2016, pp. 770-778, doi: 10.1109/CVPR.2016.90.

[7] J. S. Y. Sia, T. S. Tan, A. Yahya, M. F. T. Tiong, and J. Y. X. Sia, "Mini Kirsch Edge Detection and Its Sharpening Effect," Indonesian Journal of Electrical Engineering and Informatics, vol. 9, no. 1, pp. 228-244, 2021, doi: 10.11591/ijeei.v9i1.2597. 
[8] S. S. M. Sheet and M. S. Jarjees, "Microcontroller based in vitro hematocrit measurement system," Indonesian Journal of Electrical Engineering and Computer Science (IJEECS), vol. 18, No. 2, pp. 717-723, 2020, doi: 10.11591/ijeecs.v18.i2.pp717-723.

[9] M. Akil, Y. Elloumi, and R. Kachouri, "Detection of Retinal Abnormalitiesin Fundus Image Using Cnn Deep Learning Networks" State of the Art in Neural Networks and their Applications. Cambridge, United State: Academic Press, 2020.

[10] A. Rajan, "Detection of Diabetic Retinopathy in Fundus Image," International Journal of Science and Application, vol. 1 , no. 1, pp. 26-30, 2013.

[11] N. A. Zainudin, A. Nazari, M. M. Mustafa, W. N. W. Zakaria, N. S. Suriani, and W. N. H. W. Kairuddin, "Glaucoma detection of retinal images based on boundary segmentation," in Indonesian Journal of Electrical Engineering and Computer Science, vol. 18, no. 1, pp. 377-384, 2020, doi: 10.11591/ijeecs.v18.i1.pp377-384.

[12] J. I. Orlando, E. Prokofyeva, M. del Fresno, and M. B. Blaschko, "Convolutional Neural Network Transfer for Automated Glaucoma Identification," Proceedings Volume 10160, 12th International Symposium on Medical Information Processing and Analysis, Tandil, Argentina, 2016, doi: 10.1117/12.2255740.

[13] P. Chudzik, S. Majumdar, F. Calivá, B. Al-Diri, and A. Hunter, "Microaneurysm detection using fully convolutional neural networks," Computer Methods and Programs in Biomedicine, vol. 158, pp. 185-192, 2018, doi: 10.1016/j.cmpb.2018.02.016.

[14] J. Krause, V. Gulshan, E. Rahimy, P. Karth, K. Widner, G. S. Corrado, L. Peng, and D. R. Webster, "Grader Variability and the Importance of Reference Standards for Evaluating Machine Learning Models for Diabetic Retinopathy," Ophthalmology, vol. 125, no. 8, pp. 1264-1272, 2018, doi: 10.1016/j.ophtha.2018.01.034.

[15] Y. Jiang, H. Zhang, N. Tan, and L. Chen, "Automatic Retinal Blood Vessel Segmentation Based on Fully Convolutional Neural Networks,"in Symmetry, vol. 11, no 9, 2019, doi: 10.3390/sym11091112.

[16] C. Liu, X. Sui, Y. Liu, X. Kuang, G. Gu, and Q. Chen " Adaptive contrast enhancement based on histogram modification framework," Journal of Modern Optics, vol. 66, no. 15, pp. 1590-1601, 2019, doi: 10.1080/09500340.2019.1649482.

[17] U. Schmidt-Erfurth, A. Sadeghipour, B. S. Gerendas, S. M. Waldstein, and H. Bogunović, "Artificial intelligence in retina," Progress in Retinal and Eye Research, vol. 67, August, pp. 1-29, 2018, doi: 10.1016/j.preteyeres.2018.07.004.

[18] S. Long, J. Chen, A. Hu, H. Liu, Z. Chen, and D. Zheng, "Microaneurysms detection in color fundus images using machine learning based on directional local contrast," BioMedical Engineering OnLine, pp. 1-23, 2020, doi: 10.1186/s12938-020-00766-3.

[19] D. Le, M. Alam, C. K. Yao, J. I. Lim, Y. Hsieh, R. V. P. Chan, D. Toslak, and X. Yao, "Transfer Learning for Automated OCTA Detection of Diabetic Retinopathy," Translational Vision Science \& Technology, vol. 9, no 2, pp. 1-9, 2020, doi: 10.1167/tvst.9.2.35.

[20] X. Li, T. Pang, B. Xiong, W. Liu, P. Liang, and T. Wang, "Convolutional neural networks based transfer learning for diabetic retinopathy fundus image classification," 2017 10th International Congress on Image and Signal Processing, BioMedical Engineering and Informatics (CISP-BMEI), 2017, pp. 1-11, doi: 10.1109/CISPBMEI.2017.8301998.

[21] C. Lam, C. Yu, L. Huang, and D. Rubin, "Retinal Lesion Detection with Deep Learning Using Image Patches," Investigative Ophthalmology \& Visual Science, vol. 59, no. 1, pp. 590-596, 2018, doi: 10.1167/iovs.17-22721.

[22] M. Niemeijer, et al., "Retinopathy Online Challenge: Automatic Detection of Microaneurysms in Digital Color Fundus Photographs," in IEEE Transactions on Medical Imaging, vol. 29, no. 1, pp. 185-195, Jan. 2010, doi: 10.1109/TMI.2009.2033909.

[23] R. Asaoka et al., "Using Deep Learning and Transfer Learning to Accurately Diagnose Early-Onset Glaucoma From Macular Optical Coherence Tomography Images," American Journal of Ophthalmology, vol. 198, pp. 136145, 2019, doi: 10.1016/j.ajo.2018.10.007.

[24] S. Lu, "Accurate and Efficient Optic Disc Detection and Segmentation by a Circular Transformation," in IEEE Transactions on Medical Imaging, vol. 30, no. 12, pp. 2126-2133, Dec. 2011, doi: 10.1109/TMI.2011.2164261.

[25] X. Li, T. Pang, B. Xiong, W. Liu, P. Liang and T. Wang, "Convolutional neural networks based transfer learning for diabetic retinopathy fundus image classification," 2017 10th International Congress on Image and Signal Processing, BioMedical Engineering and Informatics (CISP-BMEI), 2017, pp. 1-11, doi: 10.1109/CISPBMEI.2017.8301998.

[26] R. Pires, H. F. Jelinek, J. Wainer, E. Valle, and A. Rocha, Advancing Bag-of-Visual-Words Representations for Lesion Classification in Retinal Images, Figshare, 2014. [Online] Available: https://figshare.com/articles/dataset/Advancing_Bag_of_Visual_Words_Representations_for_Lesion_Classification _in_Retinal_Images/953671/2

[27] M. Tsighe Hagos and S. Kant, "Transfer Learning based Detection of Diabetic Retinopathy from Small Dataset," arXiv e-prints: arXiv-1905, 2019. [Online]. Available: http://arxiv.org/abs/1905.07203

[28] G. Van Steenkiste, G. van Loon, and G. Crevecoeur, "Transfer Learning in ECG Classification from Human to Horse Using a Novel Parallel Neural Network Architecture," Scientific Reports, vol. 10, no. 1, pp. 1-12, 2020, doi: 10.1038/s41598-019-57025-2.

[29] V. Srivastava and R. K. Purwar, "Classification of eye-fundus images with diabetic retinopathy using shape based features integrated into a convolutional neural network," Journal of Information and Optimization Sciences, vol. 41, no. 1, pp. 217-227, 2020, doi: 10.1080/02522667.2020.1714186. 
[30] S. George and B. Limbasiya, "A review paper on detection and extraction of blood vessels, microaneurysms and exudates from fundus images," International Journal of Scientific \& Technology Research, vol. 2, no. 11, pp. 134-137, 2013.

[31] M. Niemeijer, B. van Ginneken, S. R. Russell, M. S. A. Suttorp-Schulten and M. D. Abràmoff, "Automated detection and differentiation of drusen, exudates, and cotton-wool spots in digital color fundus photographs for diabetic retinopathy diagnosis," National Institutes of Health, vol. 48, no. 5, pp. 2260-2267, 2007, doi: 10.1167/iovs.06-0996.

\section{BIOGRAPHIES OF AUTHORS}
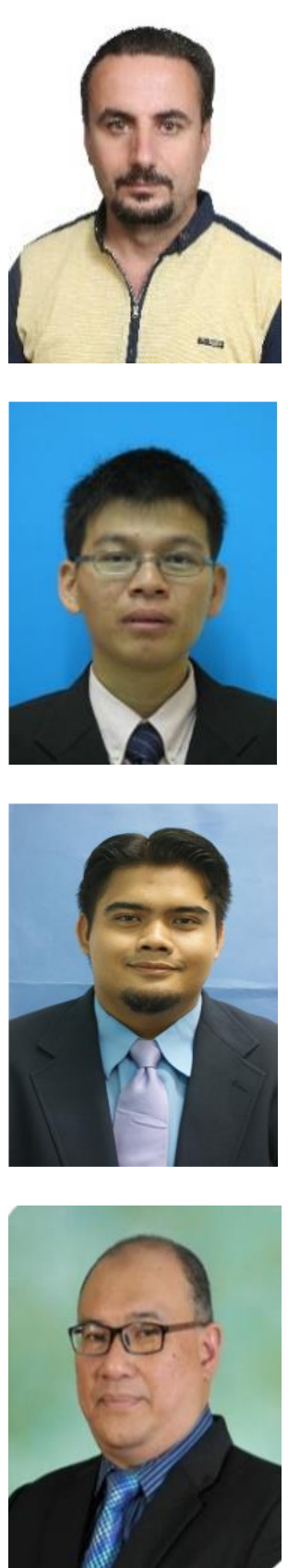

Sinan S. Mohammed Sheet received the B. Eng (Medial instrumentaion technologyEngineering) degree from technical college, Mosul, Iraq in 1999 and M.Sc. degree (Electricalcomputer and microelectronic system) in 2011 from Universiti Teknologi Malaysia (UTM), Skudai, Malaysia, and is currently pursuing the Ph.D degree in biomedical engineering at Universiti Teknologi Malaysia (UTM), Skudai, Malaysia. He is conducting the research on the image processing and deep learning for biomedical application.

Tan Tian Swee received both his M.Sc. degree and Doctoratedegree back in the year 2004 and 2008 respectively from the UniversitiTeknologi Malaysia. His research area encompasses the area of biomedical imageand signal processing and has published numerous high impact factor journals, thus establishing his expertise in the domain. He has spearheaded numerousprojects and has acquired prestigious grants from various sources and one ofhis current noTable milestones is the collaboration between UTM and IJN. He iscurrently an Associate Professor at the Faculty of Biomedical Engineering, UTM andalso serves as a Program Director in the School of Biomedical Engineeringand Health Sciences, Faculty of Engineering, UTM.

Dr. Muhammad Amir Bin As'ari holds a PhD in Biomedical Engineering from Universiti Teknologi Malaysia. His PhD's work was in the field of assistive technology, computer vision. $\mathrm{He}$ pursued his master degree and bachelor degree at Faculty of Electrical Engineering, Universiti Teknologi Malaysia, majored in Electrical Engineering. Currectly, he is working on the development of signal and image processing approaches with intelligent intervention in assistive technology and rehabilitation as well as sport performance technology especially in automated human movement recognition.

Professor Dr Wan Hazabbah Wan Hitam is the senior lecturer, consultant ophthalmologist and neuro-ophthalmologist at Department of Ophthalmology, School of Medical Sciences, Health Campus Universiti Sains Malaysia, Kubang Kerian, Kelantan, Malaysia. He obtained his MD (USM) degree in 1991 and continued his ophthalmology training at National University of Malaysia (UKM) in 1994. He received his M.Surg.(Ophthal) in the year of 1999. He was the head of department of the same centre from 2003 till 2013. He is currently the Head of Neuro-ophthalmology Service at Hospital Universiti Sains Malaysia. He was the first trained neuro-ophthalmologist in Malaysia and now the chief of Neuro-ophthalmology Subspecialty Training Programme of Malaysia. He received his fellowship training in Neuroophthalmology from The Eye Institute@ Tan Tock Seng Hospital, Singapore in 2006-2007. His main research interest areas are optic neuritis, visual pathway and visual electrophysiology. He is the first Malaysian ophthalmologist who received Tsutsui Award in 2008 from Japanese Neuro-ophthalmology Association (JANOS) for his contribution in Neuro-ophthalmology area in Asia. He was appointed as a Council Member of Asian Neuro-ophthalmology Society (ASNOS) since 2015. H 


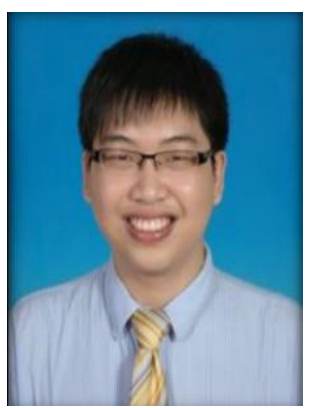

Dr. Ngoo Qi Zhe is the senior lecturer, consultant ophthalmologist. His Postgraduate (Master in Medicine - Ophthalmology) Universiti Sains Malaysia 2014-2018. Currectly, he is working on Department of Ophthalmology School of Medical Sciences Health Campus, Universiti Sains Malaysia

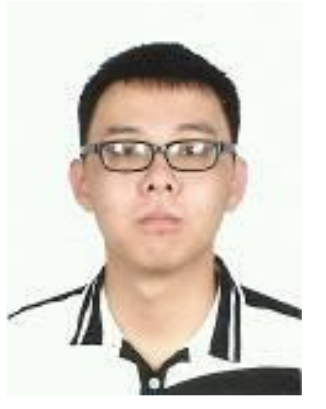

Matthias Tiong Foh Thye received the B. Eng (Electrical - Engineering) degree from Universiti Teknologi Malaysia (UTM), Skudai, Malaysia in 2018 and is currently pursuing the $\mathrm{Ph} . \mathrm{D}$ degree in biomedical engineering at Universiti Teknologi Malaysia (UTM), Skudai, Malaysia. He is conducting the research on the wireless power transfer for biomedical application. His research interests are in human tissue dielectric properties, electromagnetic safety and image processing.

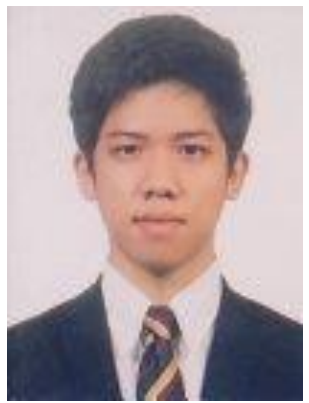

Kelvin Ling Chia Hiik received the degree of Bachelor of Engineering (ElectricalElectronics) and Master of Engineering (Electrical Power) with honours from School of Electrical Engineering, Faculty of Engineering, Universiti Teknologi Malaysia, Malaysia in 2016 and 2018 respectively. Currently, he is pursuing his Ph.D in Biomedical Engineering in School of Biomedical Engineering and Health Sciences, Faculty of Enginnering, Universiti Teknologi Malaysia, Malaysia. His research interests include signal processing, power electronics, control system and image processing. 